\title{
On the (non-)optimality of Michell structures
}

Sigmund, Ole; Aage, Niels; Andreassen, Erik

Published in:

Structural and Multidisciplinary Optimization

Link to article, DOI:

$10.1007 / \mathrm{s} 00158-016-1420-7$

Publication date:

2016

Document Version

Peer reviewed version

Link back to DTU Orbit

Citation (APA):

Sigmund, O., Aage, N., \& Andreassen, E. (2016). On the (non-)optimality of Michell structures. Structural and Multidisciplinary Optimization, 54(2), 361-373. https://doi.org/10.1007/s00158-016-1420-7

\section{General rights}

Copyright and moral rights for the publications made accessible in the public portal are retained by the authors and/or other copyright owners and it is a condition of accessing publications that users recognise and abide by the legal requirements associated with these rights.

- Users may download and print one copy of any publication from the public portal for the purpose of private study or research.

- You may not further distribute the material or use it for any profit-making activity or commercial gain

- You may freely distribute the URL identifying the publication in the public portal

If you believe that this document breaches copyright please contact us providing details, and we will remove access to the work immediately and investigate your claim. 


\title{
On the (non-)optimality of Michell structures
}

\author{
Ole Sigmund · Niels Aage · Erik Andreassen
}

Received: date / Accepted: date

\begin{abstract}
Optimal analytical Michell frame structures have been extensively used as benchmark examples in topology optimization, including truss, frame, homogenization, density and level-set based approaches. However, as we will point out, partly the interpretation of Michell's structural continua as discrete frame structures is not accurate and partly, it turns out that limiting structural topology to frame-like structures is a rather severe design restriction and results in structures that are quite far from being stiffness optimal. The paper discusses the interpretation of Michell's theory in the context of numerical topology optimization and compares various topology optimization results obtained with the frame restriction to cases with no design restrictions. For all examples considered, the true stiffness optimal structures are composed of sheets (2D) or closed-walled shell structures $(3 \mathrm{D})$ with variable thickness. For optimization problems with one load case, numerical results in two and three dimensions indicate that stiffness can be increased by up to $80 \%$ when dropping the frame restriction. For simple loading situations, studies based on optimal microstructures reveal theoretical gains of $+200 \%$. It is also demonstrated how too coarse design discretizations in $3 \mathrm{D}$ can result in un-
\end{abstract}

This work was funded by the Villum Foundation through the NextTop project.

Dept. of Mechanical Engineering, Solid Mechanics, Technical Univ. of Denmark, 2800 Kgs. Lyngby, Denmark

Tel.: +4545254256

Fax: +4545931475

E-mail: sigmund@mek.dtu.dk intended restrictions on the design freedom and achievable compliance. ${ }^{12}$

Keywords Topology optimization · Michell structures - Layout optimization · Variable thickness sheets . Optimal microstructures

\section{Introduction}

Michell's optimal frame structures (Michell, 1904) have been intensively used as benchmark problems in topology optimization. For one load case, optimized structures are often quite beautiful and consist of advanced networks of frame elements crossing each other at right angles. For aesthetic reasons Michell-like structures have also been used in architectural relations, c.f. Beghini et al (2014).

For the development of numerical tools for optimal structural design the comparison with analytical solutions based on Michell theory has been invaluable. Especially George Rozvany and co-workers (e.g. Zhou and Rozvany (1991); Rozvany (1998)) deserve a lot of honor for urging the community to benchmark numerical schemes with classical results. Michell-theory is based on fine nets of beam elements, so-called structural continua, however, in the low volume fraction limit a) optimized truss structures; b) topology optimization based on homogenization of rank- $n$ laminates (Bendsøe, 1989) and c) even generalized shape of perforated plates (Rozvany et al, 1987; Allaire and Kohn, 1993; Bendsøe and Haber, 1993) become Michell-like. Therefore, comparison with analytical solutions has become a standard

\footnotetext{
1 Preliminary results of this study were orally presented at WCSMO-11, June 7-11, 2015, Sidney, Australia.

2 This paper is dedicated to George Rozvany (1930-2015) see more in the acknowledgement.
} 
in the field and one can often read statements like "as expected, the optimized structure resembles Michelllike frame structures". Lately, similar statements have been given concerning 3D topology optimization results although analytical solutions for the 3D case are very scarce.

Michell's frame structures can be understood in a continuum sense as structures built from a 2D material with rather peculiar properties, i.e. as structures made from a material that only has stiffness in two orthogonal directions (corresponding to the principal strain directions); where the directional densities are proportional to the absolute value of the corresponding principal stress values; and who's total density is equal to the sum of the directional densities. This corresponds to an orthotopic material with zero Poisson's ratio, zero shear modulus and density proportional to the sum of the directional stiffnesses, i.e. a density twice that (or even more) of a conventional isotropic material as will be demonstrated in this paper. Such a material can directly be realized as a truss structure but is only achieved in the limit of infinitesimal local volume fraction for frame, rank $-n$ or perforated plate structures (c.f. Bendsøe and Haber (1993); Bendsøe and Sigmund (2004)). With this definition, it is obvious that one only can expect to be able to compare numerical results with the analytical solutions if one considers problems with very low volume fractions. Here volume fraction should not only be interpreted in the usual global sense, but also in a local sense where no finite local region in an optimized structure is allowed to become fully solid anywhere in the design domain.

Interestingly, all the above discussions and analytical solutions (as also mentioned in Michell's original work) assume that one is dealing with frame- or trusslike structures or perforated plates. This is also pointed out by Rozvany (1998) in connection with the advocation of Michell structures as benchmark examples: "These can be used for qualitative, and possibly quantitative, verification of discretized solutions in truss layout optimization and in generalized shape optimization of perforated plates in plane stress".

The goal of being able to compare with analytical solutions was one of the reasons for introducing the SIMP (Solid Isotropic Material with Penalization) approach. The SIMP approach ensures (almost) discrete, solid-void solutions in density-based topology optimization approaches (Zhou and Rozvany, 1991; Bendsøe, 1989). As an alternative to the penalization approach, a relaxation allowing microstructure to exist in each element (i.e. a rank- $n$ or micro-truss structure) through a homogenization approach (Bendsøe and Kikuchi, 1988) ensures point-wise 0-1 or solid-void solutions. Much later, phase-field and level-set approaches to topology optimization have aimed at solid-void designs resembling Michell structures as well. However, two interesting questions are the following: "what is the optimal structure if we don't prescribe frame-like or perforated solutions?" and the follow-up question "how much does the compliance improve for this case?". The answer to the former question is straight forward and probably not that unexpected although it seems to be largely ignored by practitioners: the optimal structure is a plateor shell-like structure of varying thickness. The answer to the latter question is highly dependent on the problem considered. In this paper practical examples considered point to improvements in stiffness of more than $80 \%$ and much more if considering (theoretically) simple loading conditions.

For 2D problems the true optimal ${ }^{3}$ structure (without the frame requirement) can be achieved by a simple interpolation model where stiffness in an element is directly proportional to the design variable, i.e. the socalled "variable thickness sheet" problem (Rossow and Taylor, 1973). It has correctly been argued that this approach does not work in 3D. Here one must use the usual power law (SIMP) approach with an exponent of 3 or more (Bendsøe and Sigmund, 1998) to get solid-void solutions that make physical sense. It turns out, however, that even in $3 \mathrm{D}$, the optimized solid-void structure is plate or shell like if the mesh is fine enough. Hence, if previously published results for 3D topology optimization were Michell-like, it is most probably either due to an on-purpose imposed minimum length-scale or due to lack of mesh refinement, i.e. an artificially imposed length scale originating in a too coarse finite element discretization.

The findings of this paper are not revolutionary and should actually be quite obvious to the theoretically well-founded readers. Nevertheless, we find it appropriate and timely to remind the community about this insight - especially considering the increasing amount of papers appearing on 3D topology optimization solutions and 3D printed micro-truss-based structures.

That optimal structures are close-walled and not open-walled (i.e. truss-like), can also be concluded from the theory of optimal composites. Here, the optimal lamination for 3D compliance minimization is a rank3 structure or a Vigdergauz type (Vigdergauz, 1994) microstructure which both are closed-walled cell structures built from sheets of varying thickness. That closed-

\footnotetext{
3 We note that the term "optimal" is grossly overused in the discussion of numerical topology optimization results in the literature, however, the variable thickness sheet problem is indeed a convex problem and hence convergence to global optima can in this case be guaranteed.
} 
walled structures are non-optimal was elaborated on also by Sigmund (1999) in connection with a discussion of the optimality of bone structure. Here it was shown that closed-walled structures are significantly stiffer than open-walled ones and hence the reason for most bone structure being open-walled must stem from other criteria than pure stiffness optimization.

This paper demonstrates its points by examples and some simplified microstructural analytical studies. Section 2 considers a 2D cantilever problem where results from variable thickness sheet, homogenization approach with optimal microstructures, SIMP approach, homogenization approach with optimal isotropic microstructures and truss topology optimization are compared to the analytical Michell solution. Section 3 considers the same cantilever problem in 3D as well as the Michell 3D torsion ball and a regular torsion rod example. Section 4 studies theoretical savings in closed-walled (sheet-like) versus open-walled (Michell-like) periodic microstructures and discusses how these results can be used to predict potential gains in stiffness for closed-walled as opposed to open-walled finite structures. Finally, Section 5 discusses the overall results and their implications for topology optimization.

\section{D cantilever study}

As a test problem in $2 \mathrm{D}$ we use a 1 by 2 cantilever fixed at its bottom ${ }^{4}$ and loaded with a unit horizontal load distributed over the central $20 \%$ of the top edge. The goal of the optimization problem is to minimize structural compliance by distributing a certain volume fraction $f$ of isotropic material within the design domain. The test problem is run with different approaches and algorithms as explained below. Common for all cases is the Young's modulus for the solid material $E_{0}=1$ and Poisson's ratio $\nu_{0}=0$ (0 to comply with the analytical solution). Objective values and optimized topologies do not change significantly for other values of Poisson's ratio. The following paragraphs briefly list background and specific settings for the various approaches.

Simplified Isotropic Material with Penalization (SIMP): Uses the simple density interpolation scheme

$E(\rho)=E_{\min }+\left(E_{0}-E_{\min }\right) \rho^{p}$

4 Note that the analytical Michell solution is pin-supported at the lower corners but this would cause stress singularities when modeled using a continuum formulation. We have found that reducing the line support to the lower three rightmost and leftmost elements, mimicking a pinned support does not change the conclusions of this study. to model the relation between element-wise constant Young's modulus and element density $\rho$. Here, $E_{\min }=$ 0.001 and $p=1$ for the variable thickness sheet case and $p=4$ for the penalized case. The basic code is the 99-line Matlab code (Sigmund, 2001). To ensure (near) optimal solutions, we use a continuation approach with a (sensitivity) filter size of 1.2 elements. The penalization is initialized to $p=1$ and gradually increased in steps of 0.2 every 50 iterations (or upon convergence) up to its final value. At the end, the filter is switched off to ensure convergence to (near) discrete solutions. With this problem formulation (and $p=4$ ) we seek the solution to the 0-1 topology optimization problem for a given mesh with no other restrictions than a minimum filter size that just avoids the checkerboard problem but does not provide mesh-independency. We note here that the variable thickness sheet problem $(p=1)$ is a convex problem and hence convergence to the global optimum is guaranteed.

Optimal microstructure: Here we use Allaire and coworkers homogenization-based, rank-2 code (downloadable from:

www.cmap.polytechnique.fr/ allaire/freefem.html).

This code is run with a continuation approach for stability reasons, starting with $\rho_{\min }=0.01$ and after 100 iterations this value is reduced to $\rho_{\min }=0.001$. With this problem formulation we seek the solution to the relaxed topology optimization problem where an optimal, infinitely fine microstructure is introduced in each element, hence ensuring existence of a solution.

Optimal isotropic microstructure: Again we use the 99line Matlab code but with the usual SIMP interpolation (1) exchanged with an interpolation scheme based on the Hashin-Strikhman upper bounds where bulk and shear moduli are interpolated independently

$$
\begin{aligned}
& \kappa(\rho)=\rho \kappa_{0}+(1-\rho) \kappa_{\min }-\frac{\rho(1-\rho)\left(\kappa_{0}-\kappa_{\min }\right)^{2}}{(1-\rho) \kappa_{0}+\rho \kappa_{\min }+\mu_{0}}, \quad(2) \\
& \mu(\rho)=\rho \mu_{0}+(1-\rho) \mu_{\min }-\frac{\rho(1-\rho)\left(\mu_{0}-\mu_{\min }\right)^{2}}{(1-\rho) \mu_{0}+\rho \mu_{\min }+\frac{\kappa_{0} \mu_{0}}{\kappa_{0}+2 \mu_{0}}},
\end{aligned}
$$

where $\kappa_{0}=E_{0} /\left(2\left(1-\nu_{0}\right)\right)$ and $\mu_{0}=E_{0} /\left(2\left(1+\nu_{0}\right)\right)$ and similar with the min values. The isotropic HashinStrikhman bounds are realized by a Vigdergauz-like triangular structure (Vigdergauz, 1999; Sigmund, 2000). This scheme is also run with $E_{\min }=0.001$ but without continuation and, as for the SIMP scheme, the filter is switched off for the final iterations. With this problem formulation we seek the solution to the relaxed topology 
Table 1 Compliance results for 2D cantilever test case

\begin{tabular}{|ll|c|c|c|c|}
\hline & $f=0.125$ & $f=0.25$ & $f=0.50$ & $f=0.75$ \\
\hline a & Thickness & 42.11 & 44.95 & 51.88 & 61.52 \\
b Rank-2 & 50.88 & 52.40 & 56.73 & 63.56 \\
c & SIMP $(p=4)$ & 54.71 & 54.55 & 58.14 & 64.28 \\
d & HS & 54.30 & 55.13 & 57.96 & 63.84 \\
\hline e TTO 8 & \multicolumn{4}{|c|}{52.04} \\
TTO 18 & \multicolumn{5}{|c|}{50.31} \\
TTO 392 & 49.39 \\
\hline Chan & \multicolumn{5}{|c|}{49.35} \\
\hline
\end{tabular}

optimization problem as for the "Optimal microstructure" approach above, however, the infinitely fine microstructure is not optimal and existence of a solution in not ensured.

Truss topology optimization: Here we use a public truss optimization code published by Martinez et al (2007). The method combines a heuristic element insertion and division strategy with gradient-based shape and topology optimization. Although no proof of optimality of resulting structures exists, the methods seems to converge towards analytical Michell solutions for a wide range of examples.

All the above approaches, except for the SIMP with $p=1$, yield solution to the $0-1$ problem, i.e. determine the distribution of material or no material that minimizes compliance. Hence the outcome of these approaches satisfy the same constraint as the Michell solution. In contrast, the SIMP $p=1$ case allows closewalled, sheet-like, non-porous $2 \mathrm{D}$ structures as a result but does not hinder porous solutions in forming. Hence, the $p=1$ case has more design freedom than the other approaches.

Analytical solution: The analytical Michell frame solution (for a point load) was first derived by Chan (1960) and the normalized compliance value can be read from a table in Graczykowski and Lewiński (2010). This value is $\Phi=C /(f a)=7.024707829^{2}=49.35$, where $a=2$ is the cantilever aspect ratio, $f$ is the volume fraction and $C$ is the compliance.

In the following we employ the five different topology optimization approaches and compare their resulting structures with the analytical solutions. The five approaches are run for various volume fractions and the resulting topologies are shown in Figure 1 and the corresponding compliance values are compared to the analytical solution in Table 1.

The truss code is run until 8, 18 and 392 bar elements have been introduced, respectively. First we note that the normalized compliance of the truss optimized structures in Figure 1e is independent on volume fraction and only depends on connectivity. The normalized

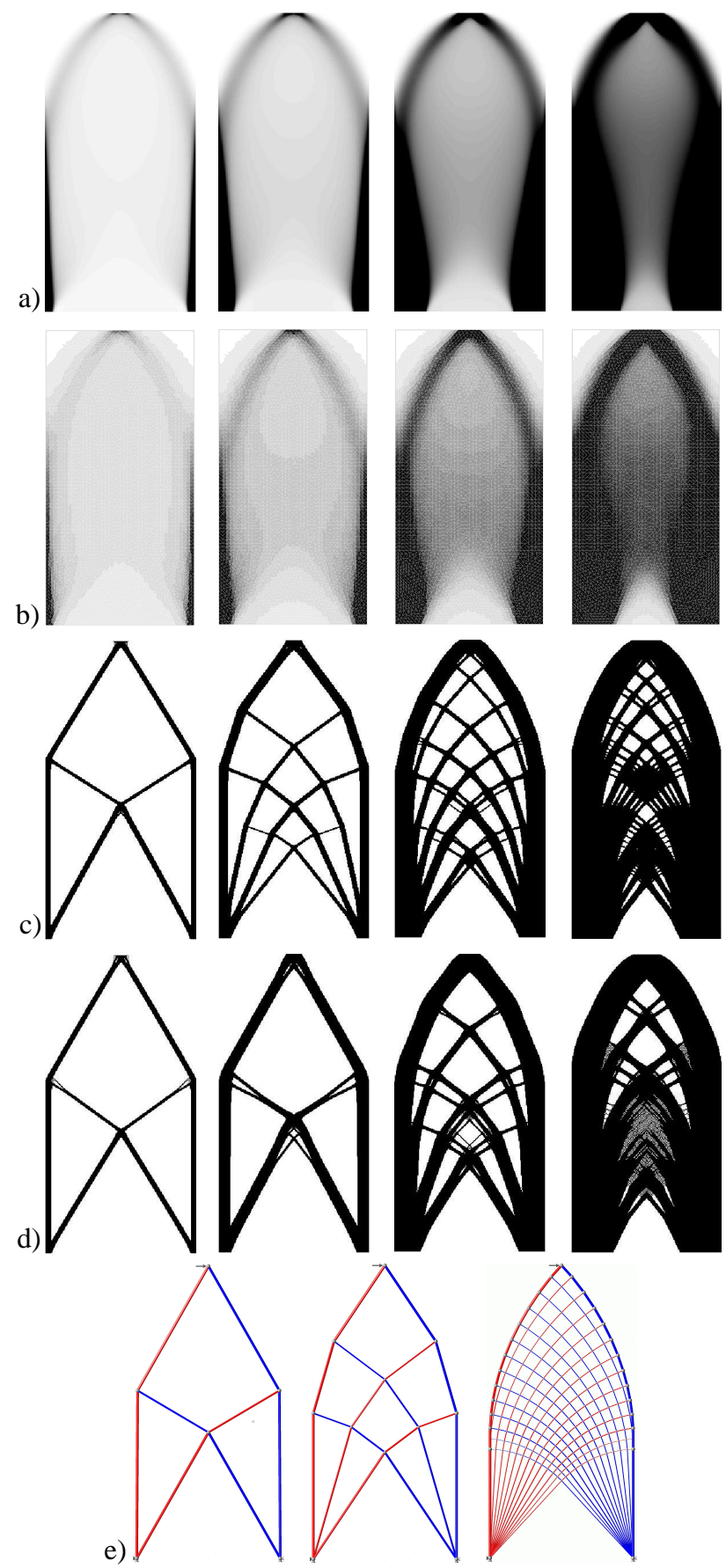

Fig. 1 Topology optimized 2D cantilever structures. a) Variable thickness sheet (SIMP with $p=1$ ), b) homogenization approach with optimal rank-2 microstructure, c) SIMP with $p=4, \mathrm{~d}$ ) optimal isotropic micro structures and e) optimized truss structures for various numbers of members denoted TTO 8, TTO 18 and TTO 392 in Table 1, respectively. 
compliance converges as expected to the Chan solution as the connectivity gets finer and finer.

The rank-2 solutions (Figure 1b) also converge to Chan's analytical solution for smaller and smaller volume fractions. When even the densest element has a density much smaller than one, the homogenizationbased solution will correspond exactly to the Michell/Chan solution, c.f. Bendsøe and Sigmund (1998).

The solutions obtained using optimal isotropic composites, i.e. using the interpolations based on the HashinStrikhman upper bounds are shown in Figure 1d. Here it is interesting to note that: a) the optimized topologies are almost entirely black-and-white indicating that the isotropy requirement actually imposes a severe penalty on intermediate densities and b) the obtained compliance values lie from $10 \%$ to $30 \%$ above the Chan solution.

The solutions obtained for penalization power $p=4$ in Figure 1c are very similar to the ones obtained for the Hashin-Strikhman interpolation (Figure 1b). This is not surprising since the physically admissible $p=4$ value for perforated composites is determined to satisfy the same Hashin-Strikhman upper bounds, c.f. Bendsøe and Sigmund (1998). It should here be noted that the number of bars in the obtained topologies is restricted by the mesh resolution. For the low volume fraction case the optimized solution corresponds to an 8-bar truss structure. Increasing the resolution from the considered 100x200 elements results in more (and extremely thin) bars, will ultimately converge to the infinitely fine Michell grid solution since no other length scale constraint than the mesh size is imposed on the problem.

Finally, we note that the normalized compliance values for the variable thickness sheet case (Figure 1a) are considerably (up to $20 \%$ ) better than the Chan solution for the small volume fraction cases and significantly better than the homogenization, SIMP and Hashin-Strikhman solutions in all cases. The most extreme case is the comparison between the variable thickness sheet solution and the $\operatorname{SIMP}(p=4)$ solution for volume fraction $f=0.125$, where the compliance of the truss-like structure is $29 \%$ higher than for the sheet solution. As for the cases above, the unconstrained problem, i.e. where no thicknesses reach the upper bound has the solution closest to the theoretical value, explaining the deterioration of normalized compliance value for higher volume fractions.

We may from this simple 2D study conclude that the frame restriction imposed by the Michell solutions results in significant reductions in achievable stiffnesses. Hence, the true compliance optimal 2D structure is a sheet-like structure with varying thickness.
Table 2 Compliance results for 3D cantilever test case with mesh refinement

\begin{tabular}{|c|c|c|c|c|c|c|}
\hline Fig. 2 & a & b & c & d & e & f \\
\hline$h$ & $1 / 16$ & $1 / 32$ & $1 / 48$ & $1 / 64$ & $1 / 80$ & $1 / 96$ \\
\hline$\Phi_{f=0.15}$ & 63.60 & 54.53 & 51.38 & 49.43 & 47.94 & 47.44 \\
\hline
\end{tabular}

\section{$33 \mathrm{D}$ studies}

In this section we extend the study to 3D. First we consider a $3 \mathrm{D}$ version of the cantilever beam studied above and thereafter we study two different 3D torsion examples.

\section{$3.13 \mathrm{D}$ cantilever study}

The first 3D example considers the same cantilever as in the previous $2 \mathrm{D}$ case but extruded $1 / 4$ in the third direction. Except for Poisson's ratio of the base material now being $\nu_{0}=0.3$ and the applied load also being distributed in the third direction everything is the same as for the $2 \mathrm{D}$ case. The volume fraction is fixed to $f=0.15$ and results and compliance values are shown in Figure 2 and Table 2, respectively. The code is a $3 \mathrm{D}$ version of the 99-line Matlab code making use of advanced multi-scale pre-conditioners as presented by Amir et al (2014). Again we use a minimum filter size of 1.2 times the element size to prevent checkerboarding and hence the only length-scale imposed is the one given by the mesh resolution. At the final iterations the filter is switched off to allow for completely black and white solutions. Figure 2a-f shows the optimized topologies for increasing mesh refinement. Resolution is refined from $16 \times 32 \times 4$ in Figure $2 \mathrm{a}$ to $96 \times 192 \times 24$ in Figure 2f. The left column shows a smoothed $3 \mathrm{D}$ view of the designs and the right column shows projected $2 \mathrm{D}$ grayscale pictures where the grayscale indicates the relative density in the out-of-plane directions. Due to symmetry in the out-of-plane direction and discreteness of the obtained 3D designs, this means that the grayscale only takes three discrete values in Figure 2a (i.e. $0,1 / 2$ or 1 corresponding to 0,2 or 4 element width in the out-of-plane direction) and 12 discrete values in Figure 2f.

Studying Figure 2 it is clear that the optimized topologies go from being truss-like for the coarse meshes to being sheet-like for the fine meshes. Also, we note that the obtained compliance values for the two finest meshes are better than the Chan value and that the compliance value of the finest mesh solution is $34 \%$ better than for the coarse mesh. 


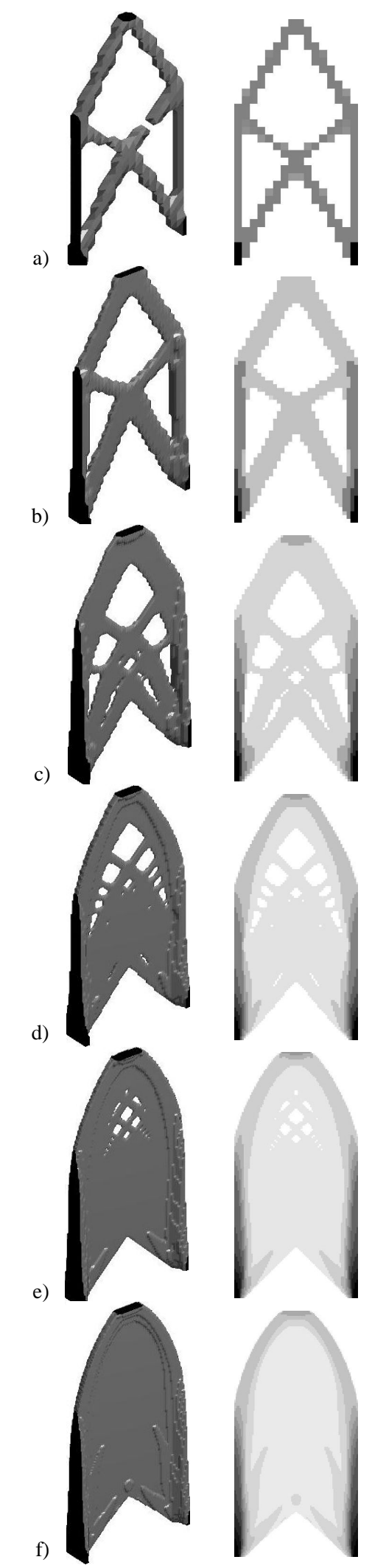

Fig. 2 Optimized cantilevers for $f=0.15$ and varying mesh refinement (from coarse a) to fine $\mathrm{f}$ )).
Table 3 Compliance results for 3D cantilever test case for varying volume fractions

\begin{tabular}{|c|c|c|c|c|c|c|}
\hline$f$ & 0.1 & 0.15 & 0.2 & 0.3 & 0.4 & 0.5 \\
\hline$\Phi$ & 53.31 & 51.38 & 50.31 & 50.30 & 52.51 & 55.14 \\
\hline
\end{tabular}

Next we fix the discretization to $48 \times 96 \times 12$ elements and vary the volume fraction. Results and compliance values are shown in Figure 3 and Table 3, respectively.

Studying Figure 3 we note that the optimized topologies go from truss-like to sheet-like with increasing volume fraction.

The two cases studied in this subsection show that optimized 3D topology is strongly dependent on mesh resolution and volume fraction. Clearly, the true optima are sheet-like structures which exhibit significantly better compliance values for the considered examples. Put in other words, if one observes Michell-like structures as result of 3D numerical topology optimization studies it is probably either due to an on-purpose introduced regularization that prevents small details (i.e. thin sheets) or large surfaces (c.f. perimeter control) or it is due to an artificial length-scale imposed by a too coarse mesh resolution.

\section{$3.23 \mathrm{D}$ torsion ball}

As the second 3D case we consider Michell's "torsion ball example". The design domain is illustrated in Figure 4 . The torsional load is here applied as point forces to a rigid part of the domain $\left(E_{\text {rigid }}=10^{3} \mathrm{E}\right.$, shown as light gray disks in Figure 4). The rigid domain is introduced to avoid stress singularities, and corresponds to applying a distributed load. Two void domains, shown as dark gray disks in Figure 4), are included to hinder material in attaching to the inner sides of the solid disks. This constraint ensures that the results can be compared to the analytical solution but has an insignificant influence on the overall design.

To solve the optimization problem, we use the topology optimization framework provided by Aage et al (2015). To achieve a black-and-white solution we extend the filtering with a projection method (Guest et al, 2004; Xu et al, 2010; Sigmund, 2007) in order to introduce a minimum length scale and hence enforce a frame-like solution.

The symmetry of the problem allows us to consider only $1 / 8$ of the domain in Figure 4 with appropriate boundary conditions. This part is discretized with $160 \times 192 \times 192 \approx 6 \mathrm{M}$ cubic elements, the volume fraction is 0.02 (excluding the passive parts of the domain), filter radius is 0.025 , threshold parameter is $\eta=0.5$, and the projection steepness parameter $\beta$ is gradually 
a)

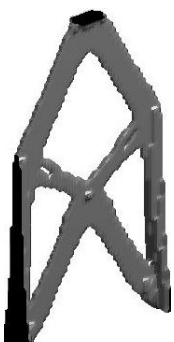

b)

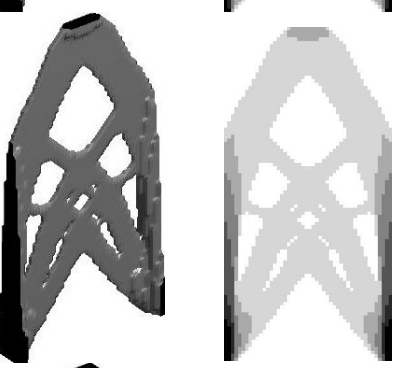

c)

d)

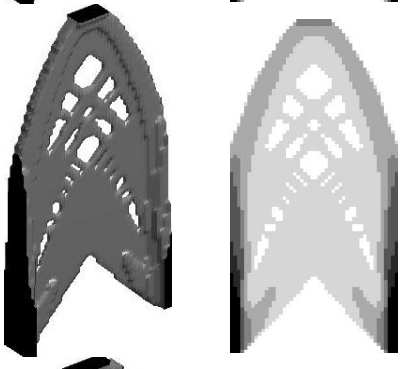

e)

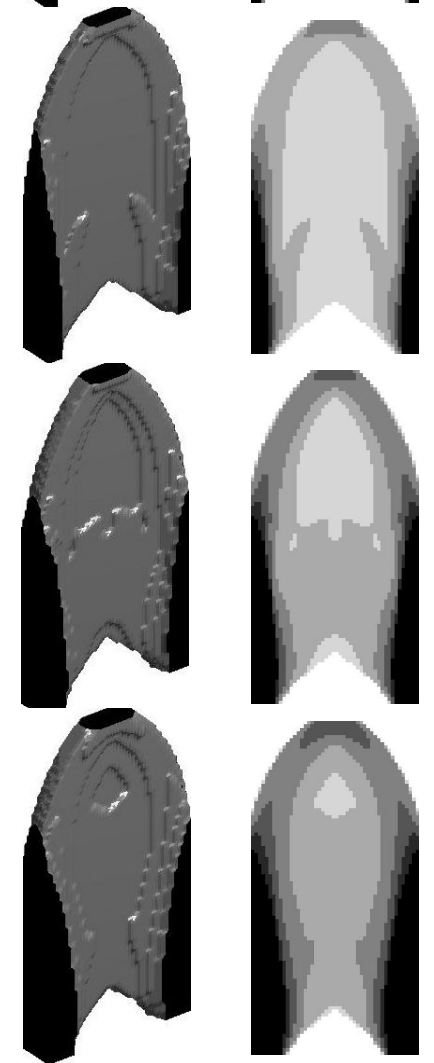

Fig. 3 3D cantilever optimized for volume fractions a)-f): $f=0.1,0.15,0.2,0.3,0.4,0.5$.

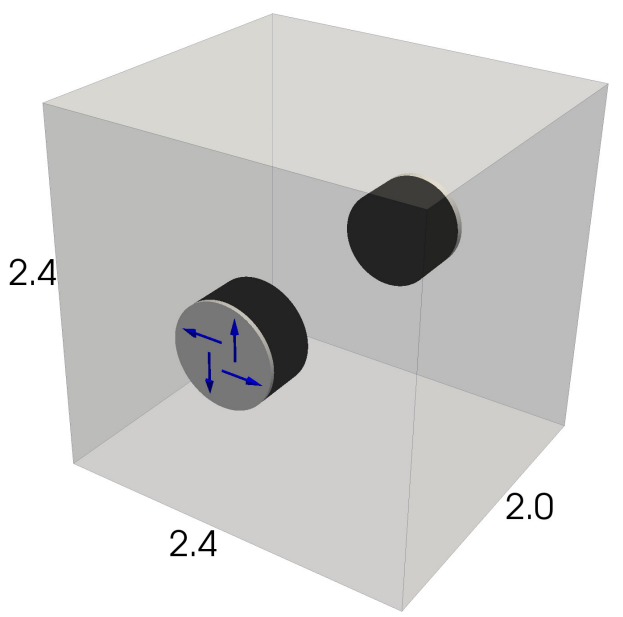

Fig. 4 Design domain for torsion ball. Blue arrows indicate loads, light gray disks represent passive, rigid parts of the domain $\left(E_{\text {rigid }}=10^{3} E\right)$ and dark gray disks indicate void domains. The disks have a radius of 0.3 and a thickness of 0.025 and 0.3 , respectively.

increased from 1 to 64 , c.f. Wang et al (2011). The resulting design is shown in Figure 5a. Interestingly, the result does not correspond to the Michell ball composed of perpendicularly crossing frame elements that one could be tempted to deduct from graphical renderings in the literature, but rather resembles a spherical closed shell which becomes thinner towards the center and thicker towards the applied torques.

The classical Michell ball, if interpreted as a coarse frame solution, can only be recovered by either using a coarse mesh that does not allow the thin shell as a solution or by enforcing a strict minimum length scale on the design. To demonstrate the latter, we rerun the example using the robust projection approach (Wang et al, 2011) with: $\eta_{d}=0.4, \eta_{i}=0.5, \eta_{e}=0.6$ and a filter radius of 0.065 . The resulting design (for $\eta=0.5$ ) recovers the spherical frame structure as a coarse scale realization of Michell's ball solution as seen in Figure 5b. Cross sections of the two spheres are compared in Figure 6 , where it can be seen how the two spheres overlap almost perfectly but are based on a thin shell and a frame structure, respectively.

The theoretically predicted compliance for the Michell ball example (Michell, 1904) is

$\Phi=\frac{16 T^{2} \log \left(\cot \left(\Theta_{0} / 2\right)\right)^{2}}{E V}$,

where $\Theta_{0}$ is the angle between the torque axis and the mid-point on the load-applying disks and $T$ is the applied torque. Inserting numbers, the analytically predicted compliance value is $255 T^{2}$, the closed sphere value is $142 T^{2}$ (for $\nu=0$ ) and $184 T^{2}$ (for $\nu=0.3$ ), whereas the compliance for the frame-like structure is 

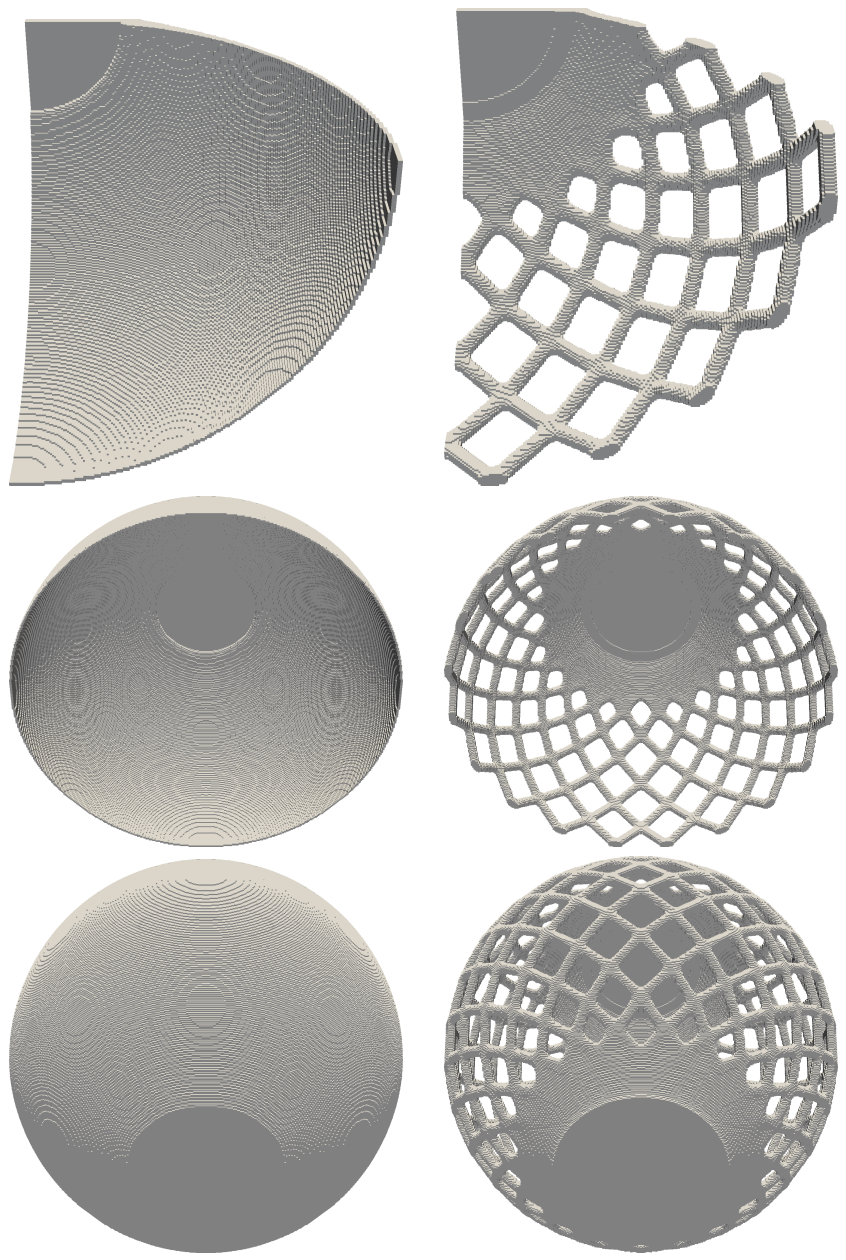

(a)

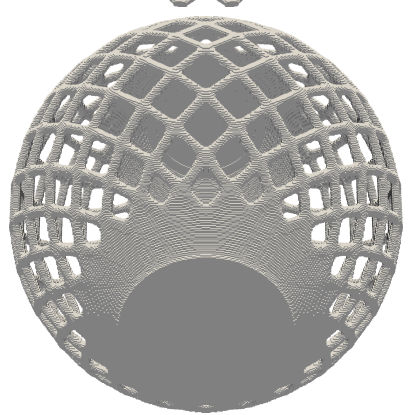

(b)

Fig. 5 Optimized designs for the problem in Figure 4, where (a) is without a minimum length scale and (b) is with a minimum length scale. The rows show - from top to bottom - an eight of, half, and the full structures.

$276 T^{2}$. Hence, the compliance value for the frame-like structure is fairly close to the analytical prediction although somewhat higher due to the rather coarse frame structure achieved and the finite volume fraction. The compliance value for the closed shell structure is close to half that of the compliance of the open structure $(\nu=0)$ and two thirds for the case of $\nu=0.3$. That is, the closed sphere is $80 \%$ stiffer than the open sphere for $\nu=0$ and $39 \%$ stiffer than the open sphere for $\nu=0.3$ ! Remark also that the numerically optimized topology is independent on the Poisson's ratio used but that the choice of Poisson's ratio does influence the obtained compliance value.

To further compare with the analytical solution we include Figure 7 where we plot the numerically obtained thickness variation of the closed shell (averaged over the ball circumference) with the analytical solution. Sup-

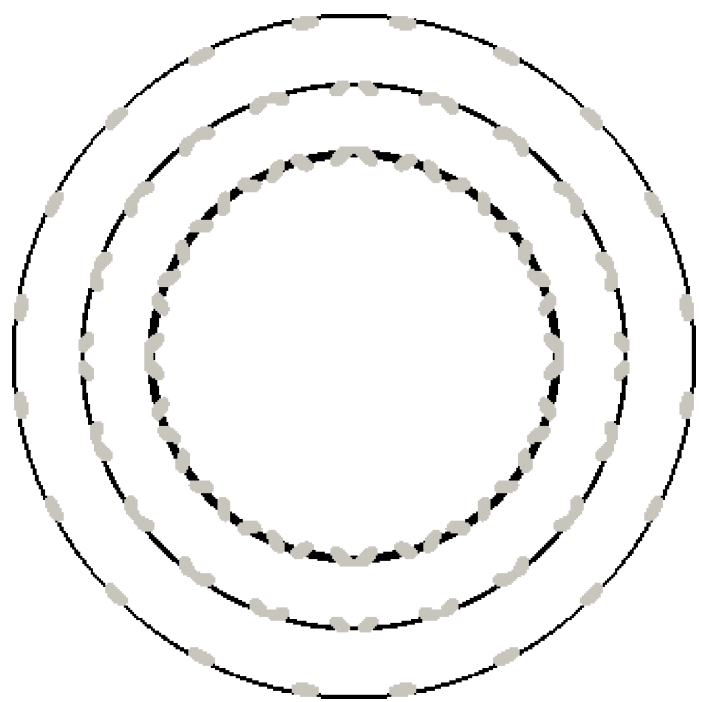

Fig. 6 Overlaid slices of the optimized designs in Figure 5 at $1 / 8,1 / 4$ and $1 / 2$ of the total sphere length. The slices of the frame structure (in gray) are overlaid the slices of the closed structure (in black).

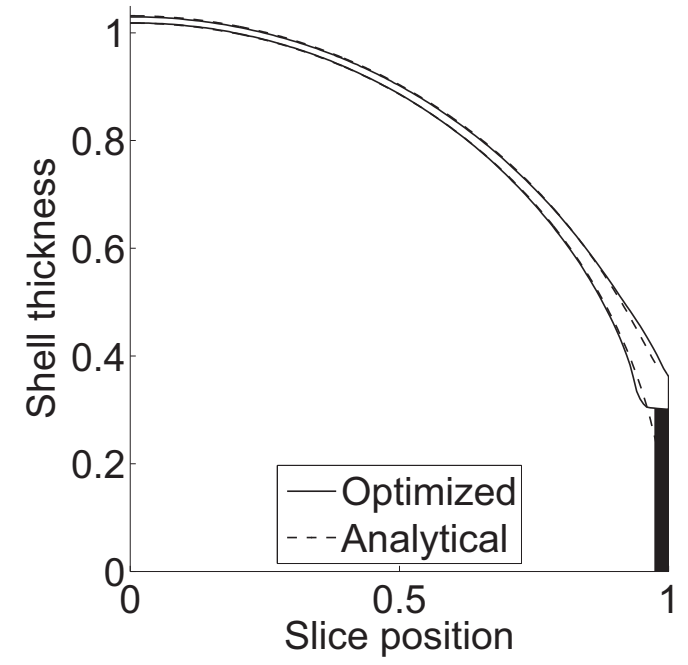

Fig. 7 Cross-sectional view of the numerically obtained thickness variation from Figure 5 compared to an analytical solution.

ported by theoretical studies, one can compare the original Michell expression for the thickness variation over the ball with an analytical solution obtained for an equishear-stressed sphere with varying thickness and made from isotropic material and get exactly the same result for the optimal thickness variation. The only difference lies in the compliance which is a factor of $2 /(1+\nu)$ higher for the peculiar Michell structural continuum compared to a simple isotropic material (see Chapter 4 for the analytical derivation of this factor). 


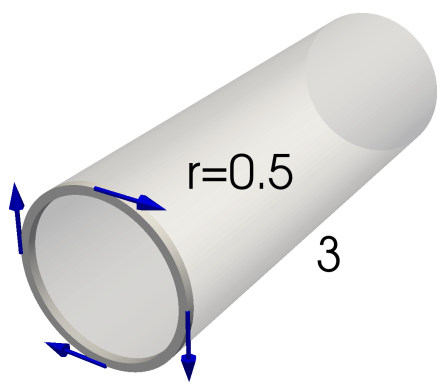

Fig. 8 Design domain for rod subject to pure torsion. Blue arrows indicate loads, and gray region represent passive, rigid part of the domain $\left(E_{\text {rigid }}=10^{3} E\right)$. The gray annulus have an outer radius of 0.5 , inner radius of 0.45 , and a thickness of 0.05. Opposite cylinder face is fully fixed.

\section{$3.33 \mathrm{D}$ torsion rod}

Finally, we consider the simple problem of optimizing a rod subject to pure torsion. The cylindrical design domain is illustrated in Figure 8, where we specify a passive, rigid part of the domain (the gray annulus) to which the torsional load is applied as four point loads but no void domains. The opposite face is fully fixed. The cylindrical domain has a radius $r=0.5$ and is discretized with 2 million cubic elements (the symmetry of the problem is not exploited). Material parameters are the same as in the previous examples and the imposed volume fraction is 0.136 .

As a reference, the theoretical compliance for a constant thickness tube with inner radius $r_{i}=0.465$ (yielding an effective volume fraction of 0.136 ) can be computed as (see e.g. Sundström, 2010)

$\Phi=\frac{T^{2} L}{J G}$,

where $T$ is the torque, $L$ is the length, $G$ is the shear modulus, and $J$ is the polar moment of inertia computed as

$J=\frac{\pi}{2}\left(r^{4}-r_{i}^{4}\right)$.

Inserting the numbers yields a theoretical compliance of $314 T^{2}$. For the Michell solution, the similar analytical compliance value is $483 T^{2}$.

Running an optimization with the above given volume constraint and without a minimum length scale constraint, using the same approach as in Section 3.2, results in the design in Figure 9a. This design is, not unexpectedly, a simple closed tube and has the compliance $326 T^{2}$, which is within a few percent of the theoretical value discussed above. Enforcing a minimum length scale using the robust approach with: $\eta_{d}=0.3$, $\eta_{i}=0.5, \eta_{e}=0.7$ and a filter radius of 0.1 , results in the design (for $\eta=0.5$ ) in Figure 9b. This design has a

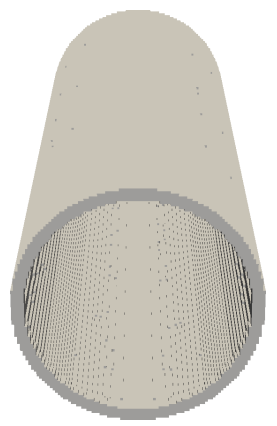

(a)

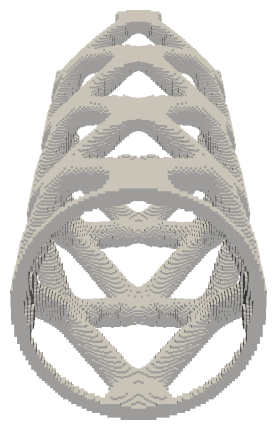

(b)
Fig. 9 Optimized designs for the problem in Figure 9, where (a) is without a minimum length scale and (b) is with a minimum length scale.
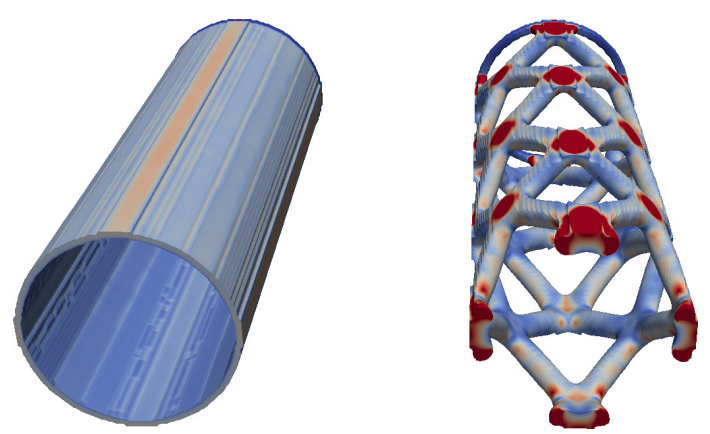

Fig. 10 Comparison of strain energy distribution in the two torsional rod designs. The color scale is the same for both designs, where blue indicates a low strain energy density and red indicates a high strain energy density.

compliance of $580 T^{2}$, which makes it far inferior to the closed tube design in Figure 9a. Figure 10 compares the strain energy distribution for the two structures.

Again, we observe a good agreement with theoretical predictions where most of the discrepancy is explained by the following. Due to the imposed length scale, the optimization is forced to put some material closer to the centerline of the design domain. In the limit, where the thickness of the tube and frame members are negligible compared to the radius of the design domain, we expect the shell design to be $54 \%$ stiffer than the frame design. The reason for this will be explained in Section 4.1.

Similar torsion examples have been studied by other researchers although for square cross sections. Nguyen et al (2012) obtained an open-walled frame-like structure which must be attributed to the coarse mesh used for the study. Villanueva and Maute (2014) studied the same problem and found that with mesh refinement the optimized structure goes from being frame-like to being closed-walled - fully in agreement with the findings of the present paper. 


\section{Optimal microstructures}

The previous sections considered structural examples with complex and spatially varying stress distributions. The considered cases demonstrated potential improvements in compliance of more than $30 \%$ when removing the Michell frame restriction and allowing thin-walled, variable-thickness-sheet-like structures. Obviously, the gains will be dependent on the local stress situations and hence one may be able to find examples where the gains are either smaller or larger. Examples of the latter will be demonstrated in the following. In particular, we will investigate the difference in stiffness of open-walled and closed-walled periodic microstructures subjected to uniform far field stresses.

The open-walled or truss-like microstructures discussed in this chapter can be seen as realizations of Michell's structural continua, i.e. materials with the peculiar properties of zero Poisson's ratio and zero shear stiffness. Similarly, the closed-walled or sheet-like microstructures correspond to the sheet like structures obtained in the previous chapters. Since analytical solutions exists for both kinds of microstructures in the small volume fraction limit, the study and comparison of these analytical results cast more light and provide exact bounds for the potential gains in dropping the frame constraint.

The search for optimal microstructure has been going on for a long time and examples of stiffness optimal structures include rank- $n$ laminates (Francfort and Murat, 1986; Lurie and Cherkaev, 1984; Milton, 1986; Norris, 1985), Hashin's coated spheres constructions (Hashin, 1962), Vigdergauz structures (Vigdergauz, 1989; Grabovsky and Kohn, 1995) and mixtures thereof (Sigmund, 2000; Gibiansky and Sigmund, 2000). All these examples consider mixtures of two or more materials and hence do not allow "variable-thicknesssheet" type solutions. This means that in 2D, optimal microstructures are restricted to perforated plate type solutions, i.e. a Michell-type restriction. In 3D, microstructures may be plate-like (c.f. Vigdergauz and rank- $n$ laminated) and hence do not suffer from the same restrictions.

In Sigmund (1999) the author discussed the optimality of bone microstructure. It had long been speculated that human bone structure is optimized with respect to the local stress situation, explaining the intricate patterns of bone structure that follow dominating stress lines. However, bone structure is mostly openwalled whereas optimal $3 \mathrm{D}$ microstructures seemed to be closed-walled. In order to investigate this discrepancy in detail, Sigmund (1999) optimized the bulk modulus of $3 \mathrm{D}$ periodic microstructures using topology op- timization and found optimized square symmetric or isotropic microstructures with bulk moduli very close to the theoretical limit. However, they were always closedwalled. Only if including a constraint for openness in the form of a conductivity constraint (mimicking the need for openness of bone structure in order to allow for transport of nutrition), the structures became openwalled. The openness, however, was achieved based on a significant sacrifice of up to $57 \%$ in the achievable bulk modulus. Hence, the study concluded that bone microstructure is not optimal with respect to stiffness alone but other factors such as e.g. permeability must play a role as well.

The conclusions by Sigmund (1999) are also relevant for the present study. Open-walled, "locally periodic Michell-like frame microstructures" are not optimal with respect to stiffness. If one allows plate-like microstructures, much stiffer material structures are achieved. The theoretically achievable stiffness improvements are studied for 2D and 3D microstructures in the following.

\subsection{Optimal 2D microstructures}

The bulk modulus for a square 2D truss microstructure can in the small volume fraction limit be found as

$\kappa_{2 D, \text { truss }}=\frac{f E}{4}$.

The Hashin-Strikhman bound for the bulk modulus of a perforated microstructure is

$\kappa_{2 D, H S}=\frac{f E}{2[(2-f(1+\nu)]}$,

which in the limit of $f \rightarrow 0$ corresponds to (7). This means that square grid (as well as triangular and hexagonal) truss and frame microstructures are optimal in the small volume fraction limit since they attain the theoretically possible bulk modulus for perforated materials.

The bulk modulus for a thin sheet is

$\kappa_{2 D, \text { sheet }}=\frac{f E}{2(1-\nu)}$.

The ratio between the bulk modulus for a thin sheet (9) and the truss microstructure (7) is

$\frac{\kappa_{2 D, \text { sheet }}}{\kappa_{2 D, \text { truss }}}=\frac{2}{1-\nu}$,

i.e. for the common Poisson's ratio of $\nu=1 / 3$ the bulk modulus of the sheet is three times bigger than for the truss microstructure! 
A similar study for the shear case gives

$\mu_{2 D, \text { truss }}=\frac{f E}{4}$

and the shear modulus for a thin sheet is

$\mu_{2 D, \text { sheet }}=\frac{f E}{2(1+\nu)}$.

The ratio between the shear modulus for a thin sheet and the truss microstructure is

$\frac{\mu_{2 D, \text { sheet }}}{\mu_{2 D, \text { truss }}}=\frac{2}{1+\nu}$,

i.e. for the common Poisson's ratio of $1 / 3$ the shear modulus of the sheet is 1.5 times bigger than for the truss microstructure.

The latter result explains the numerical observations for the torsion ball and simple torsion examples discussed in Section 3.2 and 3.3. In both these cases, the outer shell (or frame) structure is loaded in perfect shear, meaning that the expected theoretical gain for the closed shell structure should be $54 \%$ (for the Poisson's ratio of 0.3 used in the examples or $100 \%$ for the $\nu=0$ case. The ball example actually achieves gains of 39 and $80 \%$, respectively, which must be said to be quite close to the theoretical prediction when considering various disturbing factors such as finite volume fraction, coarseness of discretization and non-uniform (shear) stress distribution of the complex geometry. The simple torsion example achieves a gain of $48 \%$ which is closer to the theoretical value of $54 \%$. We are convinced that in both cases, further mesh-refinement and smaller volume fractions will make the gain numbers converge to the theoretical predictions.

\subsection{Optimal 3D microstructures}

The bulk modulus for an open-walled cubic microstructure in $3 \mathrm{D}$ can in the small volume fraction limit be found as

$\kappa_{3 D, \text { open }}=\frac{f E}{9}$

by calculating the axial stress resulting from an axial unit strain and assuming $1 / 3$ of the cell material provides stiffness as truss structure (i.e. independent on Poisson's ratio) in each direction and dividing by 3 . Likewise, the closed-walled bulk modulus can be found as

$\kappa_{3 D, \text { closed }}=\frac{2 f E}{9(1-\nu)}$

by summing axial and transversal stress from an axial unit strain and assuming $2 / 3$ of the material as a thin plate structure in each direction and dividing by 3 (and using plane stress assumption for the transversal contraction of the sheets).

The Hashin-Strikhman bound for the 3D bulk modulus is

$\kappa_{3 D, H S}=\frac{2 f E}{3[(3(1-\nu)-f(1+\nu)]}$,

which, in the limit of $f \rightarrow 0$, corresponds to (15). This means that box-like, closed-walled microstructures (and tetragonal closed-walled microstructures as well) are optimal in the small volume fraction limit since they attain the theoretically possible bulk modulus for the $3 \mathrm{D}$ bulk modulus.

The ratio between the bulk modulus for the closed (15) and the open-walled (14) microstructure is

$\frac{\kappa_{3 D, \text { closed }}}{\kappa_{3 D, \text { open }}}=\frac{2}{1-\nu}$,

i.e. for the common Poisson's ratio of $1 / 3$ the bulk modulus of the closed cell is 3 times bigger than for the open cell - the same as in the 2D case. In other words: low volume fraction truss microstructures as e.g. often used as in-fill in $3 \mathrm{D}$ printing are up to or even more than three times less efficient than their closed-walled counterparts!

\subsection{D numerical verification}

Above theoretical observations have been verified with 3D numerical homogenization (see e.g. Guedes and Kikuchi, 1990; Hassani and Hinton, 1998). For our experiments we have used a 3D numerical homogenization code very similar to the 2D homogenization code provided by Andreassen and Andreasen (2014), but utilizing the parallel solvers available in PETSc (Balay et al, 2015).

We consider a cubic unit cell and a closed-walled box microstructure with wall-thickness given by one element size. Then we consider the same unit cell but with the material confined to square cross-section frame elements at the 12 edges of the cube as illustrated in Figure 11.

Using the numerical homogenization code we calculate the effective bulk modulus of the closed and open cells for decreasing volume fractions and compare them with the theoretical bounds in Table 4. Remark, even for a mesh consisting of $120^{3}=1,728,000$ elements the lowest possible volume fraction still capable of producing a closed unit cell is around five percent (explaining the dash in the $\kappa_{\text {closed }}^{H}$-column for $\left.f=8 \cdot 10^{-4}\right)$.

From the table it is observed that the bulk modulus of the closed-walled cell structures indeed attains the theoretical bound value and that the ratio of the 

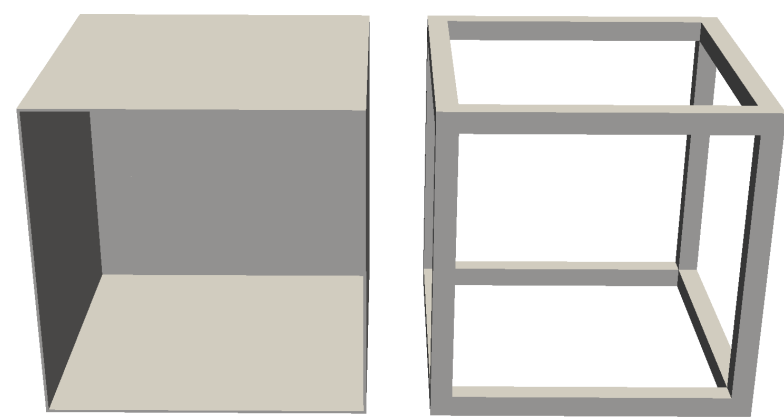

Fig. 11 Left: Closed box unit cell (thickness illustrated by showing it with front side cut off). Right: Unit cell with same amount of material in an open cell configuration.

Table 4 Results for 3D homogenization study

\begin{tabular}{|c|c|c|c|c|c|}
\hline Mesh & $f \approx$ & $\kappa_{H S}$ & $\kappa_{\text {closed }}^{H}$ & $\kappa_{\text {open }}^{H}$ & $\frac{\kappa_{\text {closed }}^{H}}{\kappa_{\text {open }}^{H}}$ \\
\hline $80^{3}$ & 0.1 & 0.0325 & 0.0324 & 0.0160 & 2.03 \\
$120^{3}$ & 0.05 & 0.0161 & 0.0160 & 0.00667 & 2.40 \\
$120^{3}$ & $8 \cdot 10^{-4}$ & $2.61 \cdot 10^{-4}$ & - & $9.40 \cdot 10^{-6}$ & 2.61 \\
\hline
\end{tabular}

bulk modulus of the closed-walled and the open-walled structures indeed approaches 3 in the low volume fraction limit as predicted by the theory in the previous subsection.

\section{Discussion}

This paper has shown how the restriction of topology optimization results to be frame-like or to be perforated plates, either by penalization in $2 \mathrm{D}$ or by use of regularization or too coarse discretizations in $3 \mathrm{D}$, results in structures that are far from being optimal with respect to pure stiffness objectives. By allowing thin sheets or closed-walled cells instead of enforcing perforated plates or open-walled microstructures gives theoretical improvements of more than $200 \%$ or more than $30 \%$ in the practical cases considered.

This conclusion holds for pure stiffness optimization. Obviously other optimization criteria or the addition of other constraint may or will change this conclusion. Some of these options are briefly discussed in the following:

- Structural stability: It is obvious that a very thin plate or sheet is more prone to buckling than a compact bar with the same material volume. Hence, buckling load criteria may favor Michell-like solutions from sheet or plate-like ones at the cost of the achievable stiffness. Ideally, buckling loads should be included in practical topology optimization. At present problems of limited size and complexity are solvable. This is partly due to the heavier computational burden of solving eigenvalue problems and partly due to huge number of multiple eigenvalues that are expected to appear for complex 3D problems.

- Microstructural stability: As for structural stability, open-walled and closed-walled microstructures are expected to behave differently with respect to buckling stability. Which are better should be studied in more detail and depends on the scale of the microstructures (since buckling loads scale non-linearly with size).

- Porosity: As for the case of bone-microstructure, other requirements than structural stiffness may favor open-walled from close-walled structures. One should keep in mind though that the imposing of open walls or perforated plates is very costly as demonstrated throughout this paper.

- Manufacturing: Very small details and thin plates and sheets may be impossible to manufacture. As demonstrated, imposing a minimum length scale, either systematically through some regularization scheme or less systematically and not recommendable through the use of too coarse meshed will results in Michell-like instead of sheet-like structures at the cost of structural stiffness.

- Transparency: Obviously, it is not relevant to look for the theoretically stiffest highrise if this implies that the structure will be closed walled without any windows. Hence, requirements to transparency will favor Michell-like instead of closed-walled stiffness optimal structures.

- Elegance/Aestatics: Michell structures are inarguably beautiful and also the very popular complete or partial micro-truss structures produced by many 3D printers look elegant and efficient. Following the recommendation in this paper will result in closed-walled, and from the outside, boring looking structures. Hence, elegance or aesthetics criteria may favor Michell-like structures and opencelled microstructures from stiffness optimal closedcell ones.

We have shown how variable thickness sheet-like or closed-walled microstructures are considerably stiffer than Michell-like and open-walled ones. Other criteria like stability, porosity, manufacturing, transparency or aesthetics may favour Michell structures to be optimal after all. However, we emphasize that the outcome of minimum compliance-type continuum topology optimization studies should always be of sheet type unless other constraints (like the afore mentioned) that favor Michell-like structures have been explicitly stated. The most general violation of this rule is seen in papers that use coarse finite element meshes and conclude that optimized 3D structures are truss-like. This is most likely 
due to the artificial length-scale imposed by the mesh and hence this (artificial and non-recommendable) type of length-scale control should be avoided or at least acknowledged when presenting algorithms and results.

Acknowledgements The authors dedicate this paper to George Rozvany (1930-2015): partly due to his life-long and great efforts in providing and promoting benchmarks to the structural optimization society; partly for valuable email exchanges concerning the benchmark examples used in this paper; and partly for his general and significant scientific and personal impact on the field of structural and multidisciplinary optimization and on the first author's scientific career in particular.

\section{References}

Aage N, Andreassen E, Lazarov B (2015) Topology optimization using PETSc: An easy-to-use, fully parallel, open source topology optimization framework. Structural and Multidisciplinary Optimization 51(3):565-572

Allaire G, Kohn RV (1993) Explicit bounds on the elastic energy of a two-phase composite in two space dimensions. Q Appl Math 51:675-699

Amir O, Aage N, Lazarov B (2014) On multigrid-cg for efficient topology optimization. Structural and Multidisciplinary Optimization 49(5):815-829

Andreassen E, Andreasen C (2014) How to determine composite material properties using numerical homogenization. Computational Materials Science 83:488-495, DOI 10.1016/j.commatsci.2013.09.006

Balay S, Abhyankar S, Adams M, Brown J, Brune P, Buschelman K, Dalcin L, Eijkhout V, Gropp W, Kaushik D, Knepley M, McInnes L, Rupp K, Smith B, Zampini S, Zhang H (2015) PETSc users manual. Tech. Rep. ANL-95/11 Revision 3.6, Argonne National Laboratory, URL http://www.mcs.anl.gov/petsc

Beghini L, Beghini A, Katz N, Baker W, Paulino G (2014) Connecting architecture and engineering through structural topology optimization. Engineering structures 59:716-726, DOI 10.1016/j.engstruct.2013.10.032

Bendsøe MP (1989) Optimal shape design as a material distribution problem. Structural Optimization 1:193202

Bendsøe MP, Haber RB (1993) The Michell layout problem as a low volume fraction limit of the perforated plate optimization problem: An asymptotic study. Struct Optim 6:263-267

Bendsøe MP, Kikuchi N (1988) Generating optimal topologies in structural design using a homogeniza- tion method. Computer Methods in Applied Mechanics and Engineering 71(2):197-224

Bendsøe MP, Sigmund O (1998) Optimering af konstruktioners topologi, form og materiale. Udstillingen

Bendsøe MP, Sigmund O (2004) Topology Optimization - Theory, Methods and Applications. Springer Verlag, Berlin Heidelberg

Chan A (1960) The design of Michell optimum structures. Cranfield College of Aeronautics

Francfort GA, Murat F (1986) Homogenization and optimal bounds in linear elasticity. Archive for Rational Mechanics and Analysis 94(4):307-334

Gibiansky LV, Sigmund O (2000) Multiphase elastic composites with extremal bulk modulus. Journal of the Mechanics and Physics of Solids 48(3):461-498

Grabovsky Y, Kohn RV (1995) Anisotropy of the Vigdergauz microstructure. Journal of Applied Mechanics 62(4):1063-1065

Graczykowski C, Lewiński T (2010) Michell cantilevers constructed within a half strip. tabulation of selected benchmark results. Structural and Multidisciplinary Optimization 42(6):869-877

Guedes JM, Kikuchi N (1990) Preprocessing and postprocessing for materials based on the homogenization method with adaptive finite element methods. Computer Methods in Applied Mechanical Engineering 83:143-198

Guest J, Prevost J, Belytschko T (2004) Achieving minimum length scale in topology optimization using nodal design variables and projection functions. International Journal for Numerical Methods in Engineering 61(2):238-254

Hashin Z (1962) The elastic moduli of heterogeneous materials. ASME Journal of Applied Mechanics 29:143-150

Hassani B, Hinton E (1998) A review of homogenization and topology optimization I-homogenization theory for media with periodic structure. Computers \& Structures 69(6):707-717

Lurie KA, Cherkaev AV (1984) G-closure of a set of anisotropically conducting media in the twodimensional case. Journal of Optimization Theory and Applications 42(2):283-304

Martinez P, Marti P, Querin O (2007) Growth method for size, topology, and geometry optimization of truss structures. Structural and Multidisciplinary Optimization 33(1):13-26, DOI 10.1007/s00158-006-00439

Michell AGM (1904) The limit of economy of material in frame structures. Philosophical Magazine 8(6):589-597

Milton GW (1986) Modelling the properties of composites by laminates. In: Ericksen JL, Kinderlehrer 
D, Kohn R, Lions JL (eds) Homogenization and Effective Moduli of Materials and Media, IMA, vol 1, Springer Verlag, pp 150-174

Nguyen T, Paulino G, Song J, Le C (2012) Improving multiresolution topology optimization via multiple discretizations. International Journal for Numerical Methods in Engineering 92(6):507-530

Norris AN (1985) A differential scheme for the effective moduli of composites. Mechanic of Materials 4:1-16

Rossow MP, Taylor JE (1973) A finite element method for the optimal design of variable thickness sheets. AIAA J 11:1566-1569

Rozvany GIN (1998) Exact analytical solutions for some popular benchmark problems in topology optimization. Structural Optimization 15(1):42-46

Rozvany GIN, Ong TG, Szeto WT, Sandler R, Olhoff N, Bendsøe MP (1987) Least-weight design of perforated elastic plates. I. International Journal of Solids and Structures 23(4):521-36

Sigmund O (1999) On the optimality of bone microstructure. In: Pedersen P, Bendsøe MP (eds) Synthesis in Bio Solid Mechanics, IUTAM, Kluwer, pp $221-234$

Sigmund O (2000) A new class of extremal composites. Journal of the Mechanics and Physics of Solids $48(2): 397-428$

Sigmund O (2001) A 99 line topology optimization code written in MATLAB. Structural and Multidisciplinary Optimization 21:120-127, DOI $10.1007 /$ s001580050176, MATLAB code available online at: www.topopt.dtu.dk

Sigmund O (2007) Morphology-based black and white filters for topology optimization. Structural and Multidisciplinary Optimization 33(4-5):401-424

Sundström B (2010) Handbook of solid mechanics. Department of Solid Mechanics, KTH

Vigdergauz SB (1989) Regular structures with extremal elastic properties. Mechanics of Solids 24:57-63

Vigdergauz SB (1994) Three-dimensional grained composites of extreme thermal properties. Journal of the Mechanics and Physics of Solids 42(5):729-740

Vigdergauz SB (1999) Energy-minimizing inclusions in a planar elastic structure with macroisotropy. Structural Optimization 17(2-3):104-112

Villanueva C, Maute K (2014) Density and level setxfem schemes for topology optimization of 3-d structures. Computational Mechanics 54(1):133-150, DOI 10.1007/s00466-014-1027-z

Wang F, Lazarov B, Sigmund O (2011) On projection methods, convergence and robust formulations in topology optimization. Structural and Multidisciplinary Optimization 43(6):767-784, DOI 10.1007/s00158-010-0602-y
Xu S, Cai Y, Cheng G (2010) Volume preserving nonlinear density filter based on heaviside funtions. Structural and Multidisciplinary Optimization 41:495-505

Zhou M, Rozvany GIN (1991) The COC algorithm, part II: Topological, geometry and generalized shape optimization. Computer Methods in Applied Mechanics and Engineering 89(1-3):309-336 